\title{
Die Evidenz der Klima- und Thalassotherapie. Ein Review
}

\author{
Angela Schuh \\ Institut für Gesundheits- und Rehabilitationswissenschaften der Ludwig-Maximilians-Universität, München, Deutschland
}

$\mathrm{V}$ on der Mitte des 19. bis in die Mitte des 20. Jahrhunderts hat die Klimatherapie z.B. in der Schweiz eine große Rolle bei Lungen- und Knochentuberkulose gespielt. In etwa zeitgleich entstand die Thalassotherapie an der französischen Atlantikküste und in Deutschland im ersten Seebad Heiligendamm. Damals lagen zahlreiche Anwendungsbeobachtungen über gute Effekte der Klimatherapie vor. Ab der zweiten Hälfte des 20. Jahrhunderts wurden die physiologischen Effekte der einzelnen Klimafaktoren sowie relevante Outcomeparameter bei bestimmten Krankheitsbildern erfasst. Es wurde in dieser Zeit auch festgestellt, dass wiederholte, serielle Anwendungen $\mathrm{zu}$ funktionellen Adaptationseffekten mit Regulationsverbesserungen in verschiedenen vegetativ gesteuerten Funktionssystemen führen können [1]. Seit mehreren Jahrzehnten ist Klima- und Thalassotherapie als therapeutisches Mittel bei Haut- und Atemwegserkrankungen, auch atopischer Genese, bekannt und wird heute als stationäre Rehabilitationsmassnahme und in Form von Mutter-KindKuren als ambulante Vorsorge- und Rehabilitationsmassnahme sowie für gesundheitsbewusste Gäste in den Kurorten durchgeführt.

\section{Methodik}

Es wurde eine systematische Recherche der Literatur der vergangenen 20 Jahre (1988-2008) in den gängigen elektronischen Datenbanken Embase, Medline, PubMed, ab dem Jahr 2000 in Standardwerken sowie in Journal Ci-
Hintergrund: Klimatherapie (am Meer: Klima- und Thalassotherapie) wird seit langer Zeit zur Behandlung zahlreicher Krankheitsbilder, insbesondere von Haut- und Atemwegserkrankungen, eingesetzt. Ziel und Fragestellung: Für welche Krankheitsbilder ist die Evidenz gesichert? Methode: Systematische Auswertung der wissenschaftlichen Literatur der gängigen Datenbanken von 1988 bis 2008. Ergebnisse: Die einzelnen Wirkfaktoren der Klimatherapie sind evident. Für die Klimatherapie von atopischen Erkrankungen (Neurodermitis, Asthma bronchiale) und Trainingsmangel im Hochgebirge sind eindeutige Daten über akute und langfristige Erfolge vorhanden. Bei der Psoriasis ist grösste Evidenz für die Therapie im Hochgebirgsklima sowie für die Klima- und Thalassotherapie am Toten Meer gegeben. Auch bei weiteren Indikationen der Klimatherapie, wie den „funktionellen" Störungen des Herz-Kreislaufsystems, sprechen die Studienergebnisse für eine anhaltende Reduzierung der Symptome, vor allem im Bereich des Hochgebirgsklimas. Für die Abhärtung an der See liegen genügend Daten vor. Trotzdem sind die therapeutischen Effekte der Klima- und Thalassotherapie noch verhältnismässig unsicher, vor allem für die am meisten bekannten Indikationen Psoriasis, Neurodermitis und Asthma bronchiale. Schlussfolgerung: Für die meisten bekannten Indikationen der Klimatherapie und zum Teil der Thalassotherapie ist Evidenz für akute und langfristige Effekte vorhanden. Voraussetzung ist die Wahl der richtigen Klimazone.

Schlüsselwörter: Klimatherapie, Thalassotherapie, Heliotherapie, Hochgebirgsklima, Seeklima

\section{Evidence of the Efficacy of Climatotherapy and Thalassotherapy - A Review}

Background: Climatotherapy (at the sea: climatotherapy and thalassotherapy) has long been considered as a treatment option for various diseases, especially chronic skin and respiratory diseases. Objective: To determine for which indications evidence-based effects have been demonstrated. Methods: Systematic analysis of the relevant publications found in the major electronic databases from 1988 until 2008. Results: The effects of single climatic factors have been proven by numerous (controlled) trials. Clear evidence has been provided for acute and long-term effects of the climatotherapeutic rehabilitation of patients with atopic diseases (atopic dermatitis, bronchial asthma), nonatopic chronic skin diseases like psoriasis and lack of high-altitude physical fitness. There is evidence for the beneficial effects of Dead Sea climatotherapy and thalassotherapy in psoriasis, with long-lasting remissions. The evidence for the efficacy of climatotherapy in patients with 'functional' cardiac and circulatory diseases is sufficient as well, especially at high altitude. Even though sufficient data are available on the improvement of physical fitness at the seaside, the therapeutic effects of climatotherapy and thalassotherapy remain relatively uncertain, especially for most of the well-known indications such as psoriasis, atopic dermatitis and bronchial asthma. Conclusion: For most of the known indications of climatotherapy and in part thalassotherapy, there is evidence for acute and long-term effects, provided the appropriate climate zone is selected.

Key words: climatotherapy, climate therapy, thalassotherapy, heliotherapy, high altitude, sea 
tation Reports und Cochrane, in den Sprachen Englisch, Deutsch, Italienisch, Spanisch und Französisch durchgeführt. Als Suchbegriffe wurden climatotherapy, climate therapy, climatic therapy, thalassotherapy sowie heliotherapy und terrain cure bzw. terrain treatment verwendet. In den Ergebnissen wurden clinical trials, reviews und controlled studies unterschieden.

\section{Atopische Erkrankungen: Neurodermitis, Asthma bronchiale, Rhinitis allergica}

Die klimatischen Faktoren haben einen signifikanten Einfluss auf Prävalenz und Verlauf der atopischen Erkrankungen: Inhalationsallergene wie Hausstaubmilben, Pollen, Schimmelpilze und Tierhaare sowie weitere klimatische Faktoren wie Luftschadstoffe oder Kaltluft wirken als Auslöser und Verschlechterungsfaktoren.

Bei der Hochgebirgsklimatherapie besteht deshalb die klimatherapeutische Strategie vor allem in Allergenvermeidung und hoher Luftreinheit. Zusätzlich sind die Reduktion der Luftfeuchtigkeit und Zunahme der UVStrahlung mit der Höhe wesentliche Elemente.

Unter den Aeroallergenen stellt die Hausstaubmilbe (v.a. Dermatophagoides pteronyssinus), die sich von menschlichen und tierischen Hautschuppen ernährt und deren Exkremente das Allergen enthalten, den wichtigsten Auslöse- bzw. Verschlimmerungsfaktor für die atopische Erkrankung dar [2,3]. Allerdings ist sie unter den klimatischen Bedingungen des Hochgebirges mit niedrigeren Temperaturen und der verringerten absoluten Luftfeuchtigkeit nicht lebensfähig. Mehrere Studien aus dem Alpenraum (Davos $1600 \mathrm{~m}$, Briancon $1350 \mathrm{~m}$, Misurina $1756 \mathrm{~m}$ ) [u.a. 4,5] haben gezeigt, dass ab ca. 1600 m Höhe (auch in den Betten) keine Hausstaubmilben mehr vorhanden sind. Schimmelpilzallergiker werden im Hochgebirge ebenfalls aufgrund der Trockenheit der Luft entlastet [6]. Auch Pollenallergiker werden durch Allergenkarenz symptomfrei. Dies zeigt u.a. eine Studie aus
Istrien bei einer Klimatherapie von Kindern mit Heuschnupfen in $800 \mathrm{~m}$ Höhe [7].

Das Fehlen bzw. verminderte Vorhandensein von Luftschadstoffen wirkt sich ebenfalls positiv auf die atopischen Erkrankungen aus. Die saubere Luft im Hochgebirge entlastet laut umweltmedizinischen Untersuchungen [z.B. 8] die entzündeten und hyperreagiblen Atemwege. Dies wurde z.B. in einer klinischen Studie mit asthmatischen Kindern [9] während Klimatherapie zwischen 810 und 1010 m gefunden. Ausserdem besteht eine direkte Korrelation zwischen Luftverschmutzung und der Prävalenz allergischer Erkrankungen und Symptome [z.B. 10]. Demnach können toxische Umweltfaktoren z.B. durch IgE und Th2-Zellen vermittelte Immunreaktionen auslösen oder ver-

\section{EASI-Score \\ Eczema Area and Severity Index \\ SCORAD-Index \\ SCORing Atopic Dermatits \\ PASI-Score \\ Psoriasis Area Severity Index \\ PTH \\ Parathormon}

stärken und zu einer Verschlechterung bzw. einem akuten Ausbruch der Atopie wie Neurodermitis führen [11]. Luftschadstoffe haben darüber hinaus nicht nur durch Modifizierung des Epithels einen direkten Einfluss auf das Immunsystem, sondern rufen auch eine Zunahme der Allergenität der relevanten Aeroallergene hervor [12].

Aufgrund der trockenen Umgebungsluft führt die gesteigerte Verdunstung des an die Hautoberfläche tretenden Schweisses zu einer Normalisierung der abnormalen Schwitzfunktion der Neurodermitiker [13] und hat einen anti-entzündlichen und juckreizhemmenden Effekt. Darüber hinaus kann eine Steigerung der Schweissproduktion erreicht werden: Neurodermitiker, die am Anfang einer 4-wöchigen Klimatherapie in 1600 m Höhe unter Belastung nicht schwitzen konnten, wiesen am Ende eine signifikant höhere Schweissproduktion und damit eine weniger starke Hautüberwärmung auf. Dieses in Richtung Gesunder veränderte Schwitzverhalten wurde noch ein halbes Jahr nach der Therapie von $38 \%$ der Patienten empfunden. Es kommt auch durch die in der Höhe verstärkte UV-Strahlung insgesamt zu einer schnelleren Abheilung des Ekzems [14]. Zusätzlich konnten positive Effekte der intensiveren Sonnenstrahlung in der Höhe auf die subjektive Befindlichkeit von Neurodermitikern gefunden werden [15].

Die klimatischen Reize des Hochgebirges werden vom Körper als positiver Stress beantwortet, was einen weiteren Aspekt des klimatherapeutischen Erfolgs bei Atopikern darstellen könnte. Darauf weisen die Ergebnisse einer Studie zur 4-wöchigen Klimatherapie bei Neurodermitikern in Davos hin, in der eine Zunahme der Kortisolkonzentration gefunden wurde [16].

Immediaterfolge sind eindeutig nachgewiesen [17]. So sind nach einem 4-6-wöchigen Rehabilitationsaufenthalt in Davos in 1600 m Höhe bei Entlassung 92\% der Patienten mit Neurodermitis und Asthma bronchiale erscheinungsfrei, 6\% leicht gebessert und nur 2\% unverändert [18]. Auch in Bulgarien bei 4-wöchiger Klimatherapie mit 2246 Patienten mit atopischer Dermatitis in 2000 m Höhe [19] wurde bei $22 \%$ eine komplette Abheilung, bei $47 \%$ eine starke Verbesserung, bei $32 \%$ eine Verbesserung und nur bei weniger als $1 \%$ keine Beeinflussung des Krankheitsbildes gefunden. Vergleichbare Ergebnisse zeigten sich in derselben Studie bei Patienten mit chronischer Urtikaria.

Auch der langfristige Erfolg der Hochgebirgsklimatherapie konnte dokumentiert werden: Bei über $6000 \mathrm{~Pa}$ tienten mit atopischem Ekzem und allergischem Asthma bronchiale wurden die Häufigkeit der Krankheitsschübe, Hautbefunde, Verbrauch topischer Kortikosteriode sowie Arbeits- und Schulausfallzeiten ein Jahr vor stationärer Aufnahme, bei Aufnahme in eine Rehabilitationsklinik in Davos, bei Entlassung nach 4 und 6 Wochen und in einem Follow-up 3, 6 und 12 Monate nach Entlassung erhoben [20]. Im Follow-up (Abb. 1) zeigte sich bei beiden Krankheitsbildern noch nach 12 Mo- 


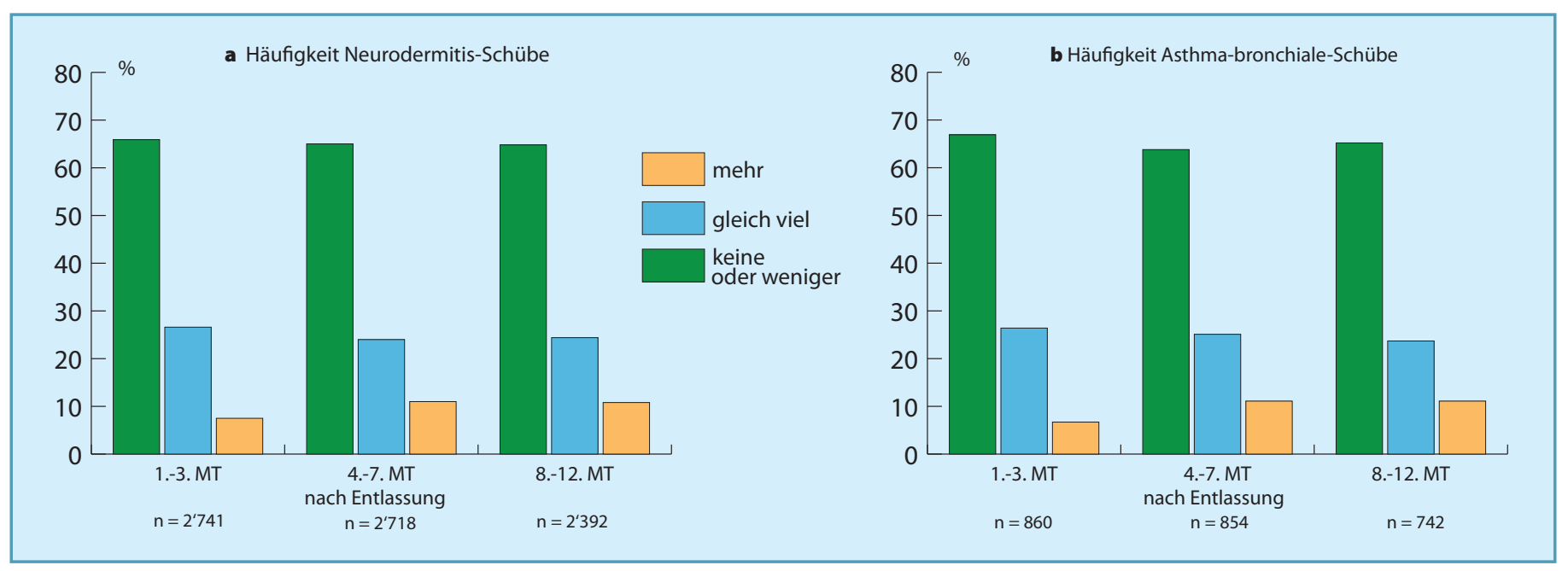

Abb. 1. Häufigkeit der Neurodermitis- (a) und Asthma-bronchiale-Schübe (b) nach Hochgebirgsklimatherapie, Follow-up bis 12 Monate [20].

naten bei zwei Drittel der Patienten eine deutliche Verbesserung. Auch der Medikamentenkonsum, vor allem der Kortikosteriodverbrauch, blieb deutlich reduziert. Die Abwesenheitszeiten in Beruf und Schule aufgrund der Erkrankung waren in den 12 Monaten nach der Hochgebirgsklimatherapie von vorher über $26 \%$ auf $2 \%$ abgesunken. In einer weiteren Studie [21] waren von 97 Patienten einer anderen Klinik in Davos bei Entlassung 89\% symptomfrei. Nach ihrer persönlichen Einschätzung hielt bei $70 \%$ der Patienten der Erfolg noch nach einem Jahr an. Zwar berichteten innerhalb des ersten Jahres $81 \%$ über einen leichten Rückfall, allerdings in deutlich geringerer Ausprägung als vor der Klimatherapie.

Auch die von den Umweltbedingungen abhängigen und häufigsten chronischen Erkrankungen im Kindesalter, extrinsic Asthma bronchiale und Neurodermitis, werden durch die Vermeidung von Allergenen bei der Klimatherapie im Hochgebirge nachweislich positiv beeinflusst [5]; so ist ein Nachlassen der bronchialen Hyperreagibilität sowie eine Verminderung von Entzündungsmarkern bei Asthma aufgrund von Höhenaufenthalten nachgewiesen [22]. Eine signifikante Reduktion der bronchialen Hyperreagibilität wurde noch 5 Monate nach Klimatherapie in $1200 \mathrm{~m}$ gefunden [23]. Eine kontrollierte Studie bestätigt den hervorragenden und längerfristig anhaltenden Effekt der Hochgebirgsklimatherapie bei Jugendlichen mit Asthma bronchiale [24]. Die untersuchten Patienten hatten eine Hausstaubmilbenallergie, wurden mit Kortikosterioden behandelt und unterzogen sich einer 10-wöchigen Kur in Davos in 1600 m Höhe. Die Ergebnisse zeigen eine signifikante Verbesserung aller gemessenen Parameter im Vergleich zu der auf Seehöhe behandelten Kontrollgruppe noch 6 Wochen nach Beendigung des Höhenaufenthaltes, während sich bei der auf Seehöhe behandelten Kontrollgruppe keine Veränderung der Parameter ergab. Somit stellen Atopien die evidenzbasierte Domäne der Hochgebirgsklimatherapie dar und führen zu langfristiger Besserung des Krankheitsbildes, was zu einem wesentlichen Teil auf die Hausstaubmilbenfreiheit zurückzuführen zu sein scheint [25,26].

Bei der Klimatherapie an der See wird ebenfalls die Strategie der Verminderung von Allergenen verfolgt. Allerdings bietet das Seeklima nur für Pollenallergiker Entlastung, da wegen der immer herrschenden hohen Luftfeuchtigkeit Hausstaubmilben und Schimmelpilze vorhanden sind. Die schadstofffreie und feuchte Luft verhindert jedoch die weitere Unterhaltung einer Schleimhautentzündung. Obwohl Asthma bronchiale eine der wichtigsten Indikationen für die Klimatherapie an Nord- und Ostsee ist [27], liegen zwar langjährige Erfahrungen [28] über ausgezeichnete Erfolge vor allem bei Kindern vor, jedoch sind bis heute nur wenige Studien, die evidenzbasierten Kriterien genügen, vorhan- den. Eine ältere Studie mit erwachsenen Patienten mit atopischer Dermatitis und Asthma bronchiale zeigte eine signifikante Beeinflussung des zellulären Status im Immunsystem durch 4wöchige Klimatherapie an der Nordsee mit einer Reduktion des IgE, einer Zunahme der Killerzellen sowie der Suppressor (T8)-Zellen, einer Reduktion der Helfer(T4)-Zellen und des T4/T8-Verhältnisses [29] und interpretiert die Ergebnisse im Sinne eines unspezifischen Effekts des Nordseeklimas. In einer weiteren Untersuchung zeigte sich neben der klinischen Verbesserung der obstruktiven Ventilationsstörung ebenfalls eine Beeinflussung der Immunlage in Form einer Zunahme von Suppressorzellen [30]. Eine multizentrische Studie zur thalassotherapeutischen Behandlung bei Neurodermitikern zwischen 3 und 65 Jahren (Klimatherapie und Meerwasserbäder, Heliotherapie) an Nord- und Ostsee [31] demonstriert eine objektive Besserung des Hautzustandes zwischen Anfang und Ende der Heilmassnahme bei mehr als 90\% der Patienten mit einer Reduktion des zur Quantifizierung eingesetzten EASIScores um im Schnitt 50\% (Abb. 2). Nur 2\% der Patienten wurden mit gleichbleibenden EASI-Werten und 5\% in einem verschlechterten Zustand entlassen. Dabei dürfte auch die nachgewiesene Normalisierung der bei Neurodermitikern gestörten Vasomotorik eine Rolle spielen [32]. So ist auch eine Normalisierung der Hautdurchblutung und der Wärmeleitfähigkeit, welche 
bei Neurodermitikern anfangs reduziert ist, feststellbar [33].

Die klima- und thalassotherapeutische Behandlung auf den Kanarischen Inseln wurde mit finnischen Jugendlichen mit schwerer atopischer Dermatitis untersucht. Sie erfuhren durch eine nur 2-wöchige Heliotherapie, kombiniert mit Meerwasserbädern eine deutliche Verbesserung: Der SCORAD Index war im Immediateffekt um 70\% reduziert und nach 3 Monaten noch immer um 45\% niedriger als vor der Therapie, der Gebrauch an topischen Steoriden war signifikant vermindert [34]. Diese unkontrollierte, prospektive Untersuchung wurde kürzlich durch eine randomisierte kontrollierte Studie mit 30 bzw. 26 norwegischen atopischen Kindern [35] bestätigt, bei der durch eine 4-wöchige Klima- bzw. Thalassotherapie auf den Kanarischen Inseln eine signifikante Verbesserung des Hautzustandes anhand des SCORAD, Zunahme der Lebensqualität und eine Verminderung des bakteriellen Hautbefalls bewiesen wurde. Da die untersuchten Kinder auch auf Hausstaubmilben reagierten und diese aufgrund der hohen Luftfeuchtigkeit auch vorhanden waren, weisen die Ergebnisse darauf hin, dass ultraviolette Strahlung und Seebäder allein bereits zu einer signifikanten Beeinflussung der atopischen Dermatitis führen können. Von grösster Wichtigkeit ist dabei wohl auch der der Einfluss der UV-B-Bestrahlung bei atopischen Patienten. Nach 2-wöchiger Heliotherapie auf den Kanaren bei Erwachsenen mit atopischer Dermatitis [36] zeigte sich neben der dadurch erzielten hochsignifikanten Verbesserung des Hautzustandes (gemessen am SCORAD) eine signifikant verbesserte Vitamin D-Balance aufgrund eines Anstiegs der SerumKalzidiol-Konzentration.

Atopische Erwachsene und Kinder werden auch am Toten Meer sehr häufig behandelt. Zwei Studien [37,38] bestätigen die ganzjährigen therapeutischen Erfolge nach einer mindestens 4 Wochen andauernden Klimatherapie mit einer Clearance in mehr als 95\% der Fälle. Für die Zeitdauer des Anhaltens der Remission werden im Durchschnitt 8 Monate angegeben. Eine Studie [39] verweist zusätzlich zur hohen

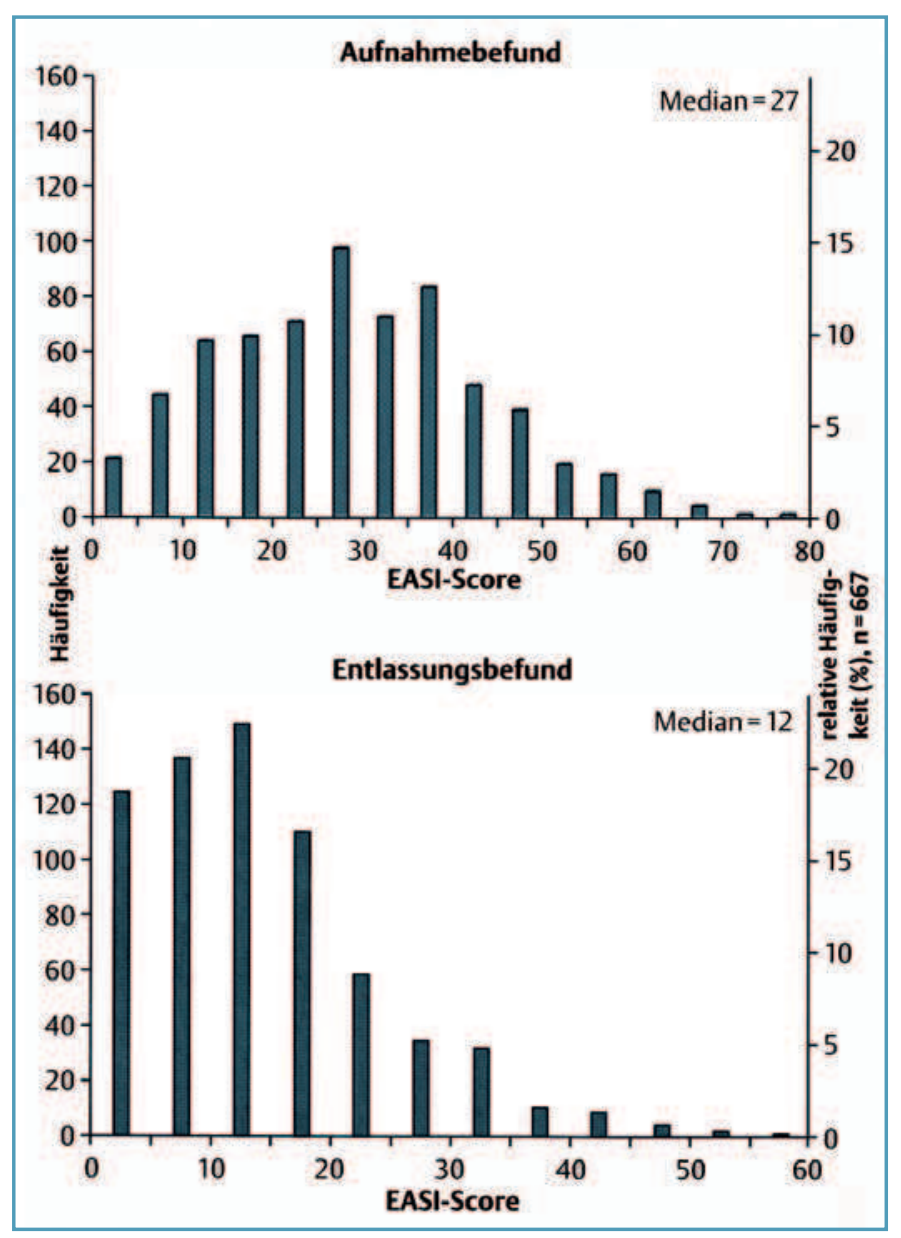

Abb. 2. Häufigkeitsverteilung der EASI-Werte am Anfang (oben) und am Ende (unten) der Therapie. Absolute Häufigkeit der Patienten (linke Ordinate), relative Häufigkeit (rechte Ordinate) [31].

UV-Strahlung auf die Effekte des hohen Magnesiumgehalts im Wasser des Toten Meeres, das Barrierefunktion, Hautfeuchtigkeit, Rauhigkeit und Entzündung beim atopischen Ekzem günstig beeinflussen soll.

\section{Nicht-allergische Atemwegserkrankungen: Bronchitis, COPD, nicht-allergisches Asthma}

Atemwegserkrankungen werden durch Luftverschmutzung ausgelöst bzw. verschlechtert. Nicht-allergisches Asthma, chronische Bronchitis und COPD (chronic obstructive pulmonary disease) sind etablierte Indikationen für die Hochgebirgsklimatherapie [10,22], saubere Luft in der Höhe und „Abhärtung“ wirken dabei durch die meist ganzjährig kühlen Temperaturen. In Mittelgebir- gen finden Patienten mit nicht-allergischen Atemwegserkrankungen aufgrund der sauberen Luft in den Wäldern ebenfalls Entlastung. Die Datenlage zu therapeutischen Effekten in beiden Klimazonen ist aber nicht ausreichend.

Bei der Klima- und Thalassotherapie an Nord- und Ostsee spielen zusätzlich zur Luftreinheit der Salzgehalt und die hohe Luftfeuchtigkeit eine Rolle. Vor allem aber wird durch die klimatischen Bedingungen eine funktionelle Adaptation des Immunsystems, die sog. „Abhärtung“ hervorrufen. Dabei ist eine direkte Beteiligung des Immunsystems an der Abhärtungsreaktion im Sinne einer verminderten Infektanfälligkeit nachgewiesen (Übersicht in SCHuH 2004]. Schon in der älteren Literatur werden die hervorragenden therapeutischen Effekte durch die Kombination der maritimen Faktoren beschrieben. An Nord- und Ostsee 
wurde mit einigen Studien gezeigt, dass durch die gezielte Abhärtung eine Verbesserung der mangelhaften thermoregulatorischen Reaktionen und Durchblutungsverhältnisse des Organismus bei Temperaturwechsel hervorgerufen wird. So fand man ein signifikant höheres Durchblutungsniveau der Nasenschleimhaut nach einer 6wöchigen Klimakur von infektanfälligen Kindern auf Sylt. Auch die konsensuelle Reaktion nach einem Kaltreiz auf die Füsse zeigte eine tendenziell kürzere Erholungszeit der Durchblutung [40]. Weitere Studien zeigen eine erhöhte Konzentration an sekretorischem IgA im Speichel von Kindern nach einer Nordsee-Klimakur [41] und einen Anstieg der Kortisolkonzentrationen, der als hormonelle Antwort auf die Klimareize im Sinne einer Abhärtung bewertet wird [42]. Bei Kindern mit (chronisch) rezidivierender Bronchitis wurde eine akute Besserung des Krankheitsbildes u.a. durch einen Anstieg der Atemstossgrösse (Einsekundenkapazität = FEV1) auf die Norm, eine Zunahme der Ausdauerleistungsfähigkeit und Reduktion der subjektiven Beschwerden gefunden [43].

\section{Hauterkrankungen: Psoriasis, Vitiligo}

Für die Behandlung der Psoriasis vulgaris sind die Sonnenbestrahlung (Heliotherapie) sowie das Baden im Meerwasser (Thalassotherapie) bzw. die Kombination aus beidem die elementaren klimatherapeutischen Bestandteile. Aufgrund zahlreicher Untersuchungen liegt heute eine Evidenz dafür vor, dass das Vitamin- $D_{3}$, das zu über $90 \%$ von der UV-B-Strahlung in der Haut gebildet wird [35], der wesentliche Vermittler für die biopositiven Effekte der Heliotherapie ist.

Im Hochgebirge wird Klimatherapie unter Ausnutzung der starken UVStrahlung bei Psoriasis, chronisch-rezidivierenden Ekzemen, chronischer Urticaria, Hautlymphomen (T-Zell-Lymphom, Mykosis fungoides), Prurigo-Erkrankungen und Akne ganzjährig eingesetzt [44] und gehört auch heute noch zu den effektivsten Behandlungsarten, gerade bei schweren Formen
[45]. So konnte am Beispiel einer Patientin mit langjähriger Psoriasis, die bereits mit verschiedensten systemischen Therapieformen behandelt wurde, nach einer 8-wöchigen Klimatherapie in Davos eine komplette Remission nachgewiesen werden [46]. Generell weisen nach mehrwöchiger Heliotherapie in Davos 95\% der Patienten bei Entlassung Erscheinungsfreiheit bzw. wesentliche Besserung des Krankheitsbildes auf; bezüglich der Rezidivfreiheit konnte eine katamnestische Untersuchung einen Zeitraum zwischen vier und sieben Monaten $(27,5 \%)$ und zwischen acht und zwölf Monaten (25,5\%) feststellen [47]. Zwei Drittel der Patienten brauchen bis $\mathrm{zu} 7$ Monate nach Hochgebirgsklimatherapie keine Kortikosteroidmedikation mehr [20,48]. Die zur Verbesserung des Krankheitsbildes benötigte UV-B-Strahlenbelastung ist während natürlicher Heliotherapie im Hochgebirge verhältnismässig gering zur Abheilung der Schuppenflechte wird während 6-wöchiger Heliotherapie in Davos nur ein 30stel derjenigen Dosis, welche die Patienten in der Regel am Wohnort mit künstlicher UV-BBestrahlung erhalten, benötigt [49].

Ein langfristiger Effekt konnte auch anhand der Einsparung von über 20\% der Behandlungskosten im Folgejahr nach 4-wöchiger Heliotherapie finnischer Patienten auf den Kanarischen Inseln festgestellt werden [50].

Auf Sonnenbestrahlung basiert auch die klima- bzw. thalassotherapeutische Behandlung der chronisch-rezidivierenden Hautkrankheiten an Nord- und Ostsee. Aufgrund der geographischen Lage sind dort therapeutisch wirksame UV-B-Intensitäten nur im Sommerhalbjahr vorhanden [51,52]. Seebäder bewirken eine Aufquellung der Haut und Lösung der krankhaft verhornten Hautschicht, die Einwirkung der Sonnenstrahlung ist danach intensiver. Klima- und Thalassotherapie an Nordund Ostsee führt erfahrungsgemäss zur deutlichen Verbesserung des Hautzustandes und lang anhaltendem Erfolg [53]. Entsprechende Patientenstudien sind jedoch nicht vorhanden. Dies betrifft nicht nur die Thalassotherapie an den deutschen Meeren, sondern generell, z.B. am Atlantik und am Mittelmeer [54]. Nur eine Untersuchung zeigt die erfolgreiche langfristige Beeinflussung der Psoriasis (PASI-Score nach 1 Woche und 2 Monaten) bei Patienten, die an kombinierter Psoriasis und arthritischer Psoriasis litten, nach Klimaund Thalassotherapie am Mittelmeer in der Türkei [55].

Die Behandlung von Hauterkrankungen wie Psoriasis und Vitiligo am Toten Meer ist dagegen durch mehrere kontrollierte Studien und Reviews gesichert. Dabei sind die UV-B-Strahlung mit hoher Intensität und einem grösseren Anteil im längerwelligen UV-BSpektrum [56,57] und generell die Vitamin- $D_{3}$-Synthese der wichtigste Wirkfaktor, Sonnenbrand ist jedoch die häufigste Nebenwirkung [58]. Der hohe Salzgehalt des Toten Meeres scheint ebenfalls einen Einfluss auf die Psoriasis zu haben [59]. Es wurden bei Psoriatikern nur aufgrund des Bades im Meer Verbesserungen im PASI-Score von immerhin $28 \%$ gefunden, bei alleiniger Sonnenexposition $72 \%$ und $83 \%$ bei kombinierter Sonnenexposition und Meeresbad [60].

Klima- und Thalassotherapie am Toten Meer führt zu einer signifikanten Verbesserung der Psoriasis, wobei nach vierwöchigem Aufenthalt von insgesamt untersuchten knapp 2000 europäischen Patienten $87 \%$ ganz oder fast (über 3/4 der Haut) symptomfrei wurden, gekoppelt mit einer deutlichen Verbesserung ihrer psychischen Verfassung. Die Remission betrug bei $74 \%$ der Patienten bis zu 6 Monate, bei jeweils 10\% 6-12 und 12-24 Monate und bei $3 \%$ der Patienten länger als 24 Monate [61]. Hervorragende Immediateffekte in ähnlicher Grössenordnung bestätigen auch alle weiteren gelisteten aktuellen Arbeiten, meist anhand des PASI-Scores [u.a. 62,63]. Die Langzeiteffekte bestätigt eine klinische Studie an 65 Psoriatikern [64]. Auch im Vergleich mit den anderen möglichen Behandlungsformen der Psoriasis scheint die Klima- und Thalassotherapie am Toten Meer insgesamt gut abzuschneiden [65], auch von der Kostenseite her [61].

Allerdings sind die Schäden durch die hohe UV-Strahlung nicht unumstritten, sowohl in Hinsicht auf die Hautalterung [66] als auch das Hautkrebsrisiko $[67,68]$. Bei einer 4-wöchigen Kli- 


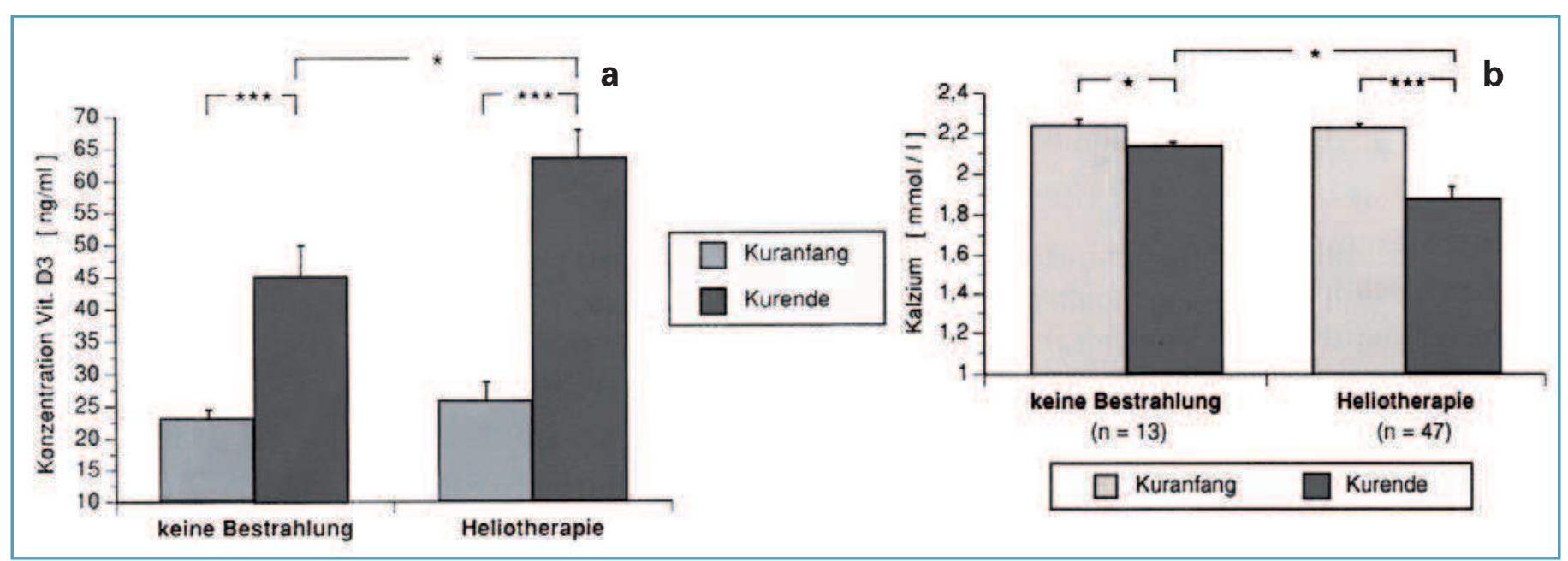

Abb. 3. Serumspiegel von Vitamin- $D_{3}(\mathbf{a})$ und Kalzium (b) vor und nach Klimatherapie (Heliotherapie) [76]

matherapie am Toten Meer erhalten die Patienten im Mittel $3,11 \mathrm{~J} / \mathrm{cm}^{2}$ an UV-B, was 148 MED entspricht [69].

Bei der Behandlung der Vitiligo scheint der hohe Mineralgehalt des Toten Meeres eine besondere Rolle $\mathrm{zu}$ spielen [70]. Nach 4-6-wöchiger Klimaund Thalassotherapie zeigten in einer klinischen Studie 11\% von über 100 behandelten Patienten eine totale Repigmentierung, $82 \%$ eine signifikante und $6 \%$ eine partielle Repigmentierung. Nur 1\% blieben unverändert. Eine katamnestische Untersuchung des medizinischen Dienstes [71] zeigte allerdings, dass die nach dem Aufenthalt am Toten Meer entstandenen Repigmentierungen nach einiger Zeit wieder verblassen. Auch bei Mycosis fungoides wurden längerfristige (5 Monate) anhaltende Erfolge publiziert [72].

In der gesamten klima- und thalassotherapeutischen Literatur konnten nur zwei Studien gefunden werden, die konstatieren, dass es keinen langfristigen Effekt der Klimatherapie bei Psoriasis gibt. So wurden bei 286 norwegischen Patienten, die sich einer 3-wöchigen Klimatherapie auf den Kanaren unterzogen, zwar signifikante Immediateffekte auf Krankheitsbild und Lebensqualität gefunden, jedoch waren nach 4 und 8 Monaten alle Messwerte wieder auf den Wert vor der Klimatherapie zurückgekehrt [73].

\section{Osteoporose}

Bei einem klimatherapeutischen Konzept zur Behandlung der Osteoporose im Hochgebirge bilden das Sonnendefizit und der Mangel an körperlicher Bewegung die klimatherapeutischen Ansatzpunkte [74,75]. Nach einer 4wöchigen Klimatherapie in Davos [76] zeigte die Heliotherapiegruppe, die während des Aufenthaltes insgesamt einer durchschnittlichen UV-B-Dosis von 41 MED (Minimale Erythemdosen) ausgesetzt war, einen signifikanten Anstieg der Vitamin- $\mathrm{D}_{3}$-Spiegel. Die Werte der Kontrolle (Gesamtdosis von im Mittel 6 MED) haben sich dagegen signifikant weniger verändert und kommen allein durch die Wirkung des UV im Freien während des Freizeitaufenthaltes zustande. Die erzielte Vitamin- $\mathrm{D}_{3}$-Zunahme liegt in einer therapeutisch relevanten Grössenordnung (Abb. 3), auch der Kalziumspiegel im Blut hat sich entsprechend verändert.

Klimatherapie von Patienten mit beginnender Osteoporose im Mittelgebirge in $800 \mathrm{~m}$ Höhe, bestehend aus Heliotherapie und Terrainkur [75], führte im Sommerhalbjahr ebenfalls bereits nach drei Wochen zu einem signifikanten Anstieg der Vitamin-D ${ }_{3}$-Produktion, einer gleichzeitigen Reduktion des Kalziumspiegels und einer leichten Zunahme der Kalzitoninkonzentration, gekoppelt mit einer geringfügigen $\mathrm{Ab}$ nahme des Parathormons im Serum. Die Vitamin- $\mathrm{D}_{3}$-Werte stiegen rund um das Dreifache an und lagen dann im oberen Viertel des Normbereiches. Die Studie weist anhand der Veränderung des Kalzitoninspiegels und des PTH auch darauf hin, dass durch die Klimatherapie ein Kalziumverlust aus dem Knochen reduziert bzw. der Wiedereinbau erhöht wird.

An Nord- und Ostsee herrschen im Sommer ebenfalls günstige Strahlungsbedingungen, um knochensubstanzfördernde Heliotherapie zu ermöglichen. Studien dazu liegen allerdings bis heute nicht vor.

\section{Prävention, 'Funktionelle' Störungen des Herz-Kreis- laufsystems ohne Organ- befund, Trainingsmangel}

Die Klimatherapie des Trainingsmangels des ganzen Körpers (Ausdauerleistungsfähigkeit und Thermoregulation) basiert auf der Erkenntnis, dass kühle Luft und Wind akut die Effekte von körperlicher Arbeit und langfristig die eines aeroben Ausdauertrainings steigern [77]. Diese sog. „Klimatische Terrainkur" führt zusätzlich zu dem durch die Bewegung ohnehin erreichten Trainingseffekt zu einem kälteinduzierten Anwachsen des aeroben Muskelstoffwechsels in einer Grössenordnung, die zu einer Verdopplung des Trainingseffekts im Vergleich zu einem Ausdauertraining bei indifferenten thermischen Bedingungen führt [27]. Die dabei eingehaltenen kühlen Körperbedingungen rufen zusätzlich eine Veränderung 
Tab. 1. Elemente der Hochgebirgsklimatherapie für Trainingsmangel und Funktionelle Herz-Kreislauferkrankungen [27]

\begin{tabular}{|c|c|}
\hline \multicolumn{2}{|c|}{$\begin{array}{l}\text { Elemente der Hochgebirgsklimatherapie für } \\
\text { „Funktionelle“ Herz-Kreislauferkrankungen }\end{array}$} \\
\hline $\begin{array}{l}\text { Klimatische Faktoren } \\
\text { - Reduzierter Sauerstoffpartialdruck } \\
\text { - Intensive Sonnenstrahlung } \\
\text { - Erniedrigte Lufttemperatur } \\
\text { - Schadstoff- und Allergenreduktion }\end{array}$ & $\begin{array}{l}\text { - } \text { Terrainkur mit kühler Köperschale } \\
\text { (Ausdauertraining + Thermoregulations- } \\
\text { training) } \\
\text { - Frischluft-Liegetherapie } \\
\text { (Entspannung, Thermoregulations- } \\
\text { training) } \\
\text { - Heliotherapie } \\
\text { (Ausdauertraining) }\end{array}$ \\
\hline $\begin{array}{l}\text { Geländefaktoren } \\
\text { Höhenterrainkurwegenetz ( } 32 \text { klassifizierte } \\
\text { Terrainkurwege mit Längen zwischen } \\
2 \text { und } 20 \text { km, in Höhenlagen zwischen } \\
1200 \text { und } 2000 \text { m), Einbeziehung steiler } \\
\text { Bergstrecken bis knapp } 3000 \text { m }\end{array}$ & $\begin{array}{l}\text { Terrainkur mit kühler Köperschale } \\
\text { - (Ausdauertraining }+ \text { Thermoregulations- } \\
\text { training) }\end{array}$ \\
\hline Thermoregulationstraining & $\begin{array}{l}\text { - Kneipp-Hydrotherapie (Arm- und Fuss- } \\
\text { bäder, Tautreten), wenn möglich im } \\
\text { Freien } \\
\text { - Schwimmen, wenn möglich in kühlem } \\
\text { Wasser }\end{array}$ \\
\hline Therapeutischer Sport & - Sportliche Gymnastik \\
\hline Dosierte Entspannung & - Entspannungsübungen \\
\hline Gesundheitsschulung & - Gesundes Leben \\
\hline $\begin{array}{l}\text { Weitere individuelle Einzelbehandlungen } \\
\text { nach Bedarf }\end{array}$ & $\begin{array}{l}\text { - z.B. Massagen } \\
\text { - Krankengymnastik } \\
\text { - Diät }\end{array}$ \\
\hline
\end{tabular}

des Fettstoffwechsels hervor: Es kommt zu einem signifikanten Abfall der Triglyceride verbunden mit einem Anstieg des Gesamt-Cholesterols bei einer Erhöhung von HDL [15].

Das Hochgebirgsklima und das Nordbzw. Ostseeklima mit ganzjährig niedrigen Lufttemperaturen und häufigem Wind bieten hervorragende Bedingungen für die Klimatherapie in Form der Terrainkur unter kühlen Bedingungen bei Patienten mit Trainingsmangel und den daraus folgenden Funktionsstörungen des Herz-Kreislaufsystems ohne Organbefund. Eine 3-wöchige Klimatherapie dieser Art (Tab. 1), in Höhenstufen zwischen $700 \mathrm{~m}$ und $3000 \mathrm{~m}$ über dem Meeresspiegel durchgeführt, erbrachte eine signifikante Verbesserung des Krankheitsbildes des Trainingsmangels [27,77]. Ausserdem ist aus höhenmedizinischen Untersuchungen bekannt, dass es aufgrund der milden Hypoxie während eines mehrwöchigen Aufenthaltes in Höhenlagen ab etwa 1'500 m zusätzlich zu einer evidenz-

\section{Koronare Herzkrankheit, Senioren}

Nach den einschlägigen Studien herrscht Konsens darüber, dass Patienten mit koronarer Herzkrankheit und ältere Menschen grundsätzlich mittlere Höhenlagen unter Beachtung der entsprechenden Vorsichtsmassnahmen aufsuchen können.

Über positive Ergebnisse der Hochgebirgsklimatherapie in $2000 \mathrm{~m}$ bei Hypertonikern (langfristige Blutdrucksenkung in Ruhe und bei körperlicher Belastung) wurde bereits früher berichtet. Bei 225 Patienten nach einem Herzinfarkt wirkte sich 3-wöchige Klimatherapie in $1600 \mathrm{~m}$ Höhe günstig auf die Blutfette aus [80]. Erst kürzlich wurden diese Ergebnisse während eines Urlaubaufenthaltes von Personen mit metabolischem Syndrom in $1700 \mathrm{~m}$ Höhe [81,82] weitgehend bestätigt. Auch in einer weiteren Untersuchung haben Patienten mit arterieller Hypertonie von einem Aufenthalt in $1500 \mathrm{~m}$ Höhe profitiert [83]. Allerdings weist auch eine Studie [84] während eines 3wöchigen klimatherapeutischen Aufenthaltes in 1660 m Höhe auf eine signifikante Reduktion des Quickwertes bzw. Verlängerung der Prothrombinzeit, d.h. auf eine verzögerte Blutgerinnung hin.

Erst kürzlich wurde bei Patienten mit Herzinsuffizienz über positive Auswirkungen einer Klimatherapie (Luftbäder in leicht kühlem Regime, Heliotherapie, Seebäder) am Schwarzen Meer auf Leistungsfähigkeit und Blutdruck berichtet, wobei die Ausprägung der Effektivität der Klimatherapie bei diesen Patienten vom Polymorphismus verschiedener Gene abhängt [85].

\section{Schlussfolgerung}

Klimatherapie bzw. Klima- und Thalassotherapie basieren auf jahrhundertealten Erfahrungen und sind in modernisierter Form heute für die Rehabilitation insbesondere von chronisch Kranken, aber auch im Sinne der Sekundärprävention und für Gesunde als Gesundheitsförderung geeignet. Dabei sind die meisten evidenzbasierten Daten zur langfristig erfolgreichen Hochgebirgsklimatherapie bei atopischen Erkrankungen und Psoriasis und 
für die Klima- und Thalassotherapie der Psoriasis am Toten Meer vorhanden. Ansatzweise sind Ergebnisse über Erfolge der Klima- und Thalassotherapie der atopischen Erkrankungen auf den Kanarischen Inseln und am Mittelmeer sowie an Nord- und Ostsee vorhanden. Vor allem jedoch für den Nachweis der Evidenz der klima- und thalassotherapeutischen Behandlung der „klassischen“ Indikation Atemwegserkrankungen an Nord- und Ostsee sind noch Studien nach den heutigen wissenschaftlich anerkannten Kriterien nötig.

\section{Literatur}

1. Gutenbrunner C: Hat die Balneologie und Medizinische Klimatologie in der Therapie chronischer Erkrankungen mehr als nur historische Bedeutung? Wien Klin Wochenschr 2006;118(9-10):251-252.

2. Disch R: High altitude climate therapy in neurodermatitis and bronchial asthma. Allergologie 1999;22(6):3-4

3. Ring J, Darsow U, Berendt H: Atopic eczeme and allergy. Curr Allergy Rep. (2001)1:39-40.

4. Razzouk $\mathrm{H}$ : Role of the environment in the onset and outcome of bronchiale asthma. Presse Thermale et Climatique 1990;127(4): 217-220.

5. Bessot JC: Climatotherapie dans l'asthma: étude critique. Rev fr Allergol 1997;37(8): 1123-1134.

6. Wagner SA: Schimmelpilze als Allergene. Allergologie 1999;22:20-25.

7. Kavuric-Hafner C: Benefits of climatotherapy in the treatment of pollinoses. Paediatria Croatica 2000;44(Suppl.1):29-31.

8. Behrendt H, Krämer U, Schäfer T, Kasche A, Eberlein-König B, Darsow U, Ring J: Allergotoxicology - a research concept to study the role of environmental pollutants in allergy. $\mathrm{ACl}$ intern 2001;13/3:122-128*.

9. Pohanka $V$, Fleischer $P$, Miskovska $M$, Trubacova D, Michnova T,'Pohnaka M et al: The role and position of the climate in complex treatment of respiratory diseases. Allergie 2007;9(4):287-291.

10. Ring J, Kramer U, Schafer T, Abeck D, Vieluf $D$, Behrendt H: Environmental risk factors for respiratory and skin atopy: Results from epidemiological studies in former East and West Germany. Int Arch Allergy Immunol 1999 Feb-Apr:118(2-4):403-407*

11. Vocks E, Engst $R$, Karl S: Dermatologic climate therapy - definition, indications and public health necessitiy. Rehabilitation 1995; 34(3):148-153

12. Behrendt $H$, Becker WM, Fritsche C, SliwaTomszok W, Tomczok J, Friedrichs KH, Ring $\mathrm{J}$ : Air pollution and allergy: experimental studies on modulation of allergen release from pollen by air pollutants. Int Arch Allergy Immunol 1997;113:69-74*

13. Schuh A Senn E: Quantifizierter Nutzen einer zusätzlichen Behandlung der Neurodermitis constitutionalis atopica durch einen mehrwöchigen Aufenthalt im Hochgebirgsklima. Z Phys Med Baln Med Klim1989;18:215-216.

14. Borelli S: Atopy and allergy: Scientific and therapeutic challenge of our time. Zeitschrift für Hautkrankheiten 1991;66 (Suppl. 2):9-19.
15. Schuh A: Climatotherapy. Experimentia 1993; 49:947-956.

16. Vocks E, Schuh A, Liebich C, Topperzer U, Ring J: Höhenaufenthalt und endogener Kortisolspiegel bei Psoriasis. Phys Rehab Kur Med 1999:197-201.

17. Vocks E, Borelli S, Rakoski J: Climatotherapy (in atopic dermatitis). Allergologie 1994;17(5): 208-213.

18. Simon D, Weigl L, Disch R: Influence of highaltitude climate therapy on atopic eczema. Allergologie 1999;22:26-28.

19. Tsankov N: High mountain climatotherapy. Clin Dermatol 1998;16:699-707.

20. Drzimalla K, Wagner SA, Disch R: Langzeitergebnisse der Hochgebirgsklimatherapie in Davos. Allergologie 1999;22:29-35.

21. Braun D, Simon D, Disch R, Schmahl FW, Borelli S: Effects of declining resources on rehabilitation of patients with chronic skin diseases. Pravention und Rehabilitation 2000, 12(1):11-19.

22. Le Roux P, Quinque K, Bonnel AS, Hastier N, Le Luyer B: Climatotherapy. Revue Francaises d'Allergologie et d'immunologie Clinique 2005;45(1):33-36.

23. Razzouk H, Faraij F, Toumi M, Le Coz J: The simultaneous development of specific bronchus reactivity and RAST in acarian subjects during a stay at altitude. Presse Thermale et Climatique 1990;127(19):37-41.

24. Grootenhorst DC, Dahlen SE, Van Den Bos JW et al.: Benefits of high altitude allergen avoidance in atopic adolescents with moderate to severe asthma, over and above treatment with high dose inhaled steroids. Clin Exp Allergy 2001;31:400-408*

25. Voack C, Rakoski J: School medical and alternative medical treatments of atopic dermatitis - Climate therapy. Allergologie 1994;17(6): 260-264

26. Golsch S, Remy W: The influence of climate therapy on patients with psoriasis vulgaris and atopic dermatitis (high altitude region versus North Sea versus dermatological clinic "without climatic factors"). Proposal for the construction of a prosepective study design. Prävention und Rehabilitation 1994;6(3):109112

27. Schuh A: Klima- und Thalassotherapie. Hippokrates, Stuttgart 2004.

28. Menger W: Klimatherapie an Nord- und Ostsee. Gustav Fischer Verlag, Jena 1997.

29. Fischer J, Schmidt-Wolf I, Rascke F: Influence of 3 weeks sojourn in the North Sea climate on subpopoulations of lymphocytes in patients with atopic dermatitis and airway disease. Z Phys med Baln Med Klim 1990; 19(6):320-324

30. Schmidt-Wolf I, Fischer J: Einfluß eines Aufenthaltes im Nordseeklima auf die Lymphozytensubpopulationen im peripheren Blut bei Patienten mit exogen allergischem Asthma bronchiale und chronischer Bronchitis. Pneumonolgie 1990;44:241-242.

31. Harms V, Buhles N, Fölster-Holst R, Gonda S, Kiosz D, Schuh H, Stick C: Therapy of atopic dermatitis at the seaside of the North Sea and Baltic Sea: therapeutic methods and quantification of the effect: A multicenter study. Phys Med Rehab Kurmed 2002;12(2): 89-94.

32. Menger W: Cold stimuli for treatment of topic dermatitis, a disease of civilisation. Phys Med Rehab Kurmed 2005;15(3):179-184.

33. Menger W: Climatotherapy: Basic principles and current significance. Intern. Prax. 2001; 41(4):911-920.

34. Autio $P$, Komulainen $P$, Larni HM: Heliotheray in atopic dermatitis: A prospective study on climatotherapy using te SCORAD index. Acta Dermato-Venerologica 20002;82(6):436-44.

35. Byremo G, Rod G, Carlsen KH: Effect of climatic change in children with atopic eczema. Allergy 2006;61:1403-1410.

36. Vähävihu K, Ylianttila L, Salmelin R, LambergAllardt C, Viljakainen H, Tuohimaa P: Heliotherapy improves vitamin $\mathrm{D}$ balance and atopic dermatitis. Brit J Dermatol 2008;158:13231328

37. Harari M, Shani J, Seidl V, Hristakieva E: Climatotherapy and atopic dermatitis at the Dead Sea: demographic evaluation and costeffectiveness. Int J Dermatol 2000;39:5969.

38. Shani J, Seidl V, Hristakieva E, Stanimirovic A, Burdo A, Harari M: Indications, contraindications and possible side-effects of climatotherapy at the Dead-Sea. Int J Dermato 1997;36(7):481-492.

39. Proksch $\mathrm{E}$, Nissen H-P, Bremgartner M, Urquhart C: Bathing in a magnesium-rich Dead Sea salt solution improves skin barrier function, enhances skin hydration, and reduces inflammation in atopic dry skin. Int $\mathrm{J}$ Dermatol 2005;44:151-157.

40. Stick C, Rischewski C, Eggert P, Scheewe S: Änderung der Nasenschleimhautdurchblutung bei infektanfälligen Kindern nach einer Klimakur an der See. Phys Rehab Kur Med 2000;10:6-10

41. Eggert P, Fay-Lorenz S, Scheewe S, Stick C: Änderung des Immunglobulins A im Speiche von Kindern nach einer Klimakur an de Nordsee. Phys Rehab Kur Med 1998;8:9-12.

42. Eggert P, Rautenberg K, Scheewe S, Stick C: Über die Veränderung der Saliva-KortisolKonzentration bei Kindern im Verlauf einer Klimakur an der Nordsee. Phys Med Rehab Kuror 2001;11(5):178-181.

43. Menger W: Physical desensitization of coldinduced bronchospasm in adolescent asthma patients. Z Phys Med Baln Med Klim 1989; 18(1):44-51

44. Engst R, Vocks E: Hochgebirgsklimatherapie bei Dermatosen und Allergien - Wirkmechanismen, Ergebnisse und Einflüsse auf immunologische Parameter. Rehabilitation 2000; 39:215-222.

45. Lebwohl M, Ting PT, Koo JYM: Psoriasis treatment: traditional therapy. Ann Rheum Dis 2005;64(Suppl.II):ii83-ii86.

46. Eichenseer M, Disch R: Climatotherapy versus systematical therapy of psoriasis. Allergologie 1999;81(1):11-19

47. Engst $R$, Fries $P$ : Klimatherapie der Psoriasis - Kritische Wertung. Hautarzt 1985;36(1):5458

48. Vocks E: Solar phototherapy in dermatological high-altitude climatotherapy. Allergologie 1999;22(6):15-19

49. Vocks E, Seifert B, Hahn H, Fröhlich C Quantitative Erfassung der Heliotherapie be Psoriasis vulgaris im Hochgebirgsklima. Z Hautkrh 1989;64:466-472.

50. Snellman E, Maljanen T, Aromaa A, Reunanen $A$, Jyrkinen-Pakkasvirta $T$, Luoma J: Effect of heliotherapy on the cost of psoriasis. Br J Dermatol 1998 Feb;1:38(2):288-292

51. Stick C, Harms V, Pielke L: Spectral masurements of solar ultraviolet radiation on onset of the North Sea. Phys Med Rehab Kur Med 1996;(1):1-6.

52. Stick C, Pielke L: The composition of solar UV-radiation during the course of the day Aktuelle Dermatologie 1998;24(6):159-163.

53. Menger W: Indikationen und Erfolg der Klimatherapie bei Kindern. Offentl. Gesundheitswesen 1989;51:9 
54. Lucchetta MC, Monaco G, Valenzi VI, Russo MV Campanella J, Nocchi S, Mennuni G: Le basi storico-scientifiche della talassoterapia: stato dell arte. Clin Ter 2007;158(6):533-541.

55. Mork C, Ozek M, Wahl AK: Treatment or leisure? Climate therapy of patients with psoriasis nd psoriasis arthritis. Tidsskrift for the Norske Laegeforening 2004;12(1):60-62.

56. Kragballe K, Avrach WW, Politit Y, Landau M, Brenner S: Climatotherapy at the Dead Sea stimulates vitamin $D_{3}$ metabolism. Venerologica 1996;76(4):324-325.

57. Kudish Al, Abels D, Harari M: Ultraviolett radiation properities as applied to photoclimateherapy at the Dead Sea. Int J Dermatol 2003;42(5):359-365.

58. Even-Paz Z: Skin cancer and climatotherapy in psoriasis. Comment. Brit J Dermatol 2001; 202.

59. Wolf R, Orion E, Matz H: Climatotherapy: There is life in the Dead Sea. IMAJ 2003;5: 124-125.

60. Even-Paz Z, Gumon R, Kipnis V, Abels DJ, Efron D: Dead Sea sun versus Dead Sea water in treatment of psoriasis. J Dermatol Treatment 1996;7(2):83-86.

61. Shani J, Harari M, Hristakieva E, Seidel V, BarGiyora J: Dead Sea climatotherapy versus other modalities of treatment for psoriasis: comparative cost-effectiveness. Int J Dermatol 1999;38:252-262.

62. Cohen $A D$, Van Dijk D, Naggan L, Vardy DA Effectiveness of climatotherapy at the Dead Sea for psoriasis vulgaris: A community-oriented study introducing the "Beer Sheva Psoriaisis Severity Score". J Dermatol Treatm 2005;16:308-313.

63. Hodak E, Gottlieb AB, Segal T, Politi Y, Maron L, David M: Climatotherapy at the Dead Sea is a remititive therapy for psoriasis: combined effects on epidermal and immunologic activation. J Am Acad Dermatol 2003 Sep;49(3): 451-457.

64. Harari M, Novack L, Barth J, David M, Friger M, Shimon WM: The percentage of patients achieving PASI 75 after 1 month and remission time after climatotherapy at the Dead Sea. Int J Derm 2007;46:1087-1091.

65. Gambichler T, Altmeyer P, Hoffmann K: Correspondence: Cost effectiveness of DeadSea climatotherapy and balneophototherapy of psoriasis. Int J Dermatol 2001;40:158-160.

66. Paltiel O, Adler B, Herschko K, Tsukrov B, David M: Are patients with psoriasis susceptible to the classic risk factors for actinic keratoses? Arch Dermatol 2004;140:805-810.

67. Frentz G, Olsen JH, Avrach A: Malignant tumors and psoriasis: climatotherapie at the Dead Sea. Brit J Dermatol. 1999;141:10881091.

68. David M, Tsukrov B, Adler B, Hershko K, Pavlotski F Rozenman D et al: Actinic dam- age among patients with psoriaisis treated by climatotherapy at the Dead Sea. J Am Acad Dermatol 2005,March;52(3):445-450.

69. Kushelevsky AP, Harari M, Kudish Al, Hristakieva E, Ingber A, Shani J: Saftey of solar phototherapy at the Dead Sea. J Am Acad Dermatol 1998;38:447-52

70. Schallreuter KU, Moore J, Behrens-Williams S, Panske A, Harari M: Rapid initiation of repigmentation in Vitiligo with Dead Sea climatotherapy in combination with pseudocatatlase (PC-KUS). Int J Dermatol 2002;41: 482-487.

71. Blick U: Katamnestische Untersuchungen zur Behandlung der Vitiligo am Toten Meer. Präv Rehab 1994;6/3:105-108.

72. Hodak E, Gottlieb AB, Segal T, Maron L, Lotem M, Feinmesser M, David M: An open trial of climatotherapy at the dead sea for patch-stage mycosis fungoides. J Am Acad Derm 2004;51(1):33-38.

73. Wahl AK, Mork C, Cooper BA, Padilla G: No long-term changes in psoriasis severity and qualitiy of life following climate therapy. J Am Acad Derm 2005;52(4):699-701.

74. Tomczak H, Hawryluk D: The role of spa treatment in the management of osteoporosis. Family Medicine and Primary Care Review 2007;9(3):877-879

75. Schuh $A$ : Vitamin- $D_{3}-$ Spiegel und weitere Knochenstoffwechselparameter im Serum von Patienten mit beginnender Osteoporose nach Heliotherapie (Sonnenbestrahlung) und künstlicher UVB-Strahlung. Phys Rehab Kur Med 1995;5:109-114.

76. Schuh A, Kneist W, Philipona R: Heliotherapie im Hochgebirge mit einer quantifizierten Strahlendosis: Einfluß auf den Vitamin-DSpiegel von Psoriasispatienten. Phys Rehab Kur Med 1995;5:21-24.

77. Schuh A: Diseases caused by lack of training and their treatment by means of climate therapy. Fortschr Medizin 1989;107(22):472.

78. Schuh A, Kneist W, Schmitt HJ: Steigerung der Ausdauerleistungsfähigkeit von durchschnittlich trainierten Personen durch natürliche Sonnenstrahlung (Heliotherapie). Phys Rehab Kur Med 1993;3:95-99.

79. Stick C, Gretz M: Physische Aktivität und Verbesserung der Ausdauerleistungsfähigkeit während einer Rehabilitationsmaßnahme an der Nordsee. Phys Rehab Kur Med 1996;6: 98-104.

80. Mirrakhimov MM, Aitbaev KA, Murataliev TM, Kim NM: Potentialities of correcting atherogenic dyslipoproteinemias with mountain climate therapy. Kardiologiya 1991;31(3): 8-10.

81. Schobersberger W, Schmid P, Lechleitner $M$, von Duvillard SP, Hortnagl ' $H$, Gunga HC, Klingler A, Fries D, Kirsch K, Spiesberger R, Pokan R, Hofmann P, Hoppichler F, Riedmann
G, Baumgartner H, Humpeler E: Austrian moderate altitude study 2000 (AMAS 2000). The effects of moderate altitude $(1700 \mathrm{~m})$ on cardiovascular and metabolic variables in patients with metabolic syndrome. Eur J Appl Physiol Feb 2003;88(6):506-514*.

82. Gunga HC, Fries D, Humpeler E, Kirsch K, Boldt LE, Koralewski E, Johannes B, Klingler A, Mittermayr M, Rocker L, Yaban B, Behn C Jelkmann W, Schobersberger W: Austrian moderate altitude study (AMAS 2000) - fluid shifts, erythropoesis and angiogenesis in patients with metabolic syndrome at moderate altitude (1700 m). Eur J Appl Physiol. 2003 88:497-505*

83. Skudlik C, Schöne D, Disch R: Klimatherapie: Wirkung auf andere Organsysteme am Beispiel des Herz-Kreislaufsystems. Allergologie 1999;22:12-14.

84. Schuh A, Götz S, Elsner P, lliev D, Keist W Senn E: Der Einfluss von mittleren Höhenlagen (1000-3000 m) auf die Blutgerinnung eine Pilotstudie. Phys RehabKur Med 1999 1:14-19

85. Kovlen DV, Tishakov A Yu, Glotov OS, Moskalenko MV, Demin GS, Bitsadze AN et al: Genetic determinants of the efficiency of climatotherapy in patients with chronic heart failure. Bulletin of Experimental Biology and Medicine 2007;143(1):26 -31.

* in den Datenbanken unter anderen Schlag wörtern gelistete Studien: (high) altitude $[24,81,82]$, environment and allergy, atopy $[10,12]$.

\section{Disclosure Statement}

The author declares that no financial or other conflict of interest exists in relation to the content of this article.

\section{Korrespondenzadresse}

Prof. Dr. Dr. med. Angela Schuh Institut für Gesundheits- und Rehabilitationswissenschaften Ludwig-Maximilians-Universität Marchioninistrasse 17, DE-81377 München angela.schuh@med.uni-muenchen.de 